\title{
QUATERNIONIC WAVE PACKETS
}

\author{
Stefano De Leo ${ }^{1}$ and Gisele C. Ducati ${ }^{2}$ \\ 1 Department of Applied Mathematics, University of Campinas \\ PO Box 6065, SP 13083-970, Campinas, Brazil \\ deleo@ime.unicamp.br \\ 2 Department of Mathematics, University of Parana \\ PO Box 19081, PR 81531-970, Curitiba, Brazil \\ ducati@mat.ufpr.br
}

Submitted: November, 2006. Accepted: April, $200 \%$.

\begin{abstract}
We compare the behavior of a wave packet in the presence of a complex and a pure quaternionic potential step. This analysis, done for a gaussian convolution function, sheds new light on the possibility to recognize quaternionic deviations from standard quantum mechanics.
\end{abstract}

Subject Classification: 03.65.Fd - 03.65.Nk (PACS).

\section{INTRODUCTION}

This article represents the third work of the authors on the Schrödinger equation in the presence of a quaternionic potential step. In the first paper [1], we have shown that, for such a potential, it is possible to calculate an analytic plane wave solution. This represents, to the best of our knowledge, the first case in which an analytic solution has been given for a quaternionic quantum mechanical potential problem (previous plane wave studies, regarding the barrier [2-4] and well [5] potentials, required numerical calculations). The possibility to work with an analytic plane wave solution allowed, in our second paper [6], a detailed discussion of the quaternionic diffusion through the stationary phase method. The motivation to write a third paper on this topic is mainly due to the old wish of the authors to find qualitative differences between complex and quaternionic formulation of quantum mechanics which could be useful in identifying the evidence of quaternionic potentials in the case in which such potentials really exist. In this spirit, the quaternionic diffusion, at the moment discussed from a general point of view by using the stationary phase method, should be investigated by directly analyzing the motion of the incident, reflected and transmitted wave packets in a potential step problem. The numerical results of this study can be then interpreted by looking for analytical approximations of the incident, reflected and transmitted wave packets in the case of gaussian convolution functions. The comparison between the complex and pure quaternionic case for the potential step seems to be the best starting point to analyze qualitative differences between quaternionic and complex quantum mechanics. Indeed, more complicated potentials can often been seen as successive potential steps. For example, a potential barrier can be successful studied as a two-step problem $[7,8]$. The investigation proposed in this paper could also give a final answer to the old question concerning the possibility to fit a pure quaternionic potential by a complex one a consequently to never recognize quaternionic deviations from standard quantum mechanics.

\section{PLANE WAVE ANALYSIS}

Before to give the explicit plane wave solution for a pure quaternionic potential step, let us show how, by a simple re-phasing of the wave function, we can transform, without lost of generalization, the 
$(j, k)$-part of the quaternionic potential

$$
i V_{1}+j V_{2}+k V_{3}
$$

into a pure $j$-part, i.e.

$$
i V_{1}+j \sqrt{V_{2}^{2}+V_{3}^{2}}
$$

This will greatly simplify our presentation. Let us begin by considering the quaternionic Schrödinger equation [9]

$$
i \frac{\hbar^{2}}{2 m} \Phi_{x x}(x, t)-\left(i V_{1}+j V_{2}+k V_{3}\right) \Phi(x, t)=\hbar \Phi_{t}(x, t) .
$$

This partial differential equation can be reduced, by using the well-know separation of variables

$$
\Phi(x, t)=\varphi(x) \exp [-i E t / \hbar],
$$

to the following second order ordinary differential equation [10]

$$
i \frac{\hbar^{2}}{2 m} \varphi^{\prime \prime}(x)-\left(i V_{1}+j V_{2}+k V_{3}\right) \varphi(x)=-\varphi(x) E i .
$$

By multiplying (from the left) the previous equation by an unitary complex number $e^{i \alpha}$ and re-writing the pure quaternionic part of the potential as

$$
j V_{2}+k V_{3}=j \sqrt{V_{2}^{2}+V_{3}^{2}} e^{-i \theta},
$$

where $\theta=\arctan \left[V_{3} / V_{2}\right]$, we find

$$
e^{i \alpha}\left[i \frac{\hbar^{2}}{2 m} \varphi^{\prime \prime}(x)-\left(i V_{1}+j \sqrt{V_{2}^{2}+V_{3}^{2}} e^{-i \theta}\right) \varphi(x)\right]=-e^{i \alpha} \varphi(x) E i .
$$

By observing that $e^{i \alpha} j=j e^{-i \alpha}$, we can rewrite the previous equation as follows

$$
i \frac{\hbar^{2}}{2 m}\left[e^{i \alpha} \varphi(x)\right]^{\prime \prime}-i V_{1}\left[e^{i \alpha} \varphi(x)\right]-j \sqrt{V_{2}^{2}+V_{3}^{2}}\left[e^{-i(\alpha+\theta)} \varphi(x)\right]=-\left[e^{i \alpha} \varphi(x)\right] E i
$$

Consequently, the choice $\alpha=-\theta / 2$ conduces to

$$
i \frac{\hbar^{2}}{2 m} \psi^{\prime \prime}(x)-\left(i V_{1}+j \sqrt{V_{2}^{2}+V_{3}^{2}}\right) \psi(x)=-\psi(x) E i
$$

where

$$
\psi(x)=e^{-i \frac{\theta}{2}} \varphi(x) .
$$

It is important to observe that constant phases paly no role in the stationary phase method and, thus, such a re-phasing has no physical effect on the motion of the incident, reflected and transmitted wave packets. For the diffusion problem, $E>V_{0}$, we shall compare the complex case, $V_{0}=V_{1}\left(V_{2}=V_{3}=0\right)$, with the pure quaternionic case, $V_{0}=\sqrt{V_{2}^{2}+V_{3}^{2}}\left(V_{1}=0\right)$.

\section{II.A COMPLEX POTENTIAL STEP}

By setting $V_{1}=V_{0}$ and $V_{2}=V_{3}=0$ in Eq.(5), we find the standard Schrödinger equation,

$$
i \frac{\hbar^{2}}{2 m} \psi_{c}^{\prime \prime}(x)-i V_{0} \psi_{c}(x)=-\psi_{c}(x) E i
$$

whose analytic plane wave solution is given by [11]

$$
\begin{array}{ll}
\text { [I] fREe REGion }(x<0): & \psi_{c, I}(\epsilon, x)=\exp [i \epsilon x]+r_{c}(\epsilon) \exp [-i \epsilon x], \\
\text { [II] POtential REgion }(x>0): & \psi_{c, I I}(\epsilon, x)=t_{c}(\epsilon) \exp [i \sigma x],
\end{array}
$$

where

$$
\epsilon=\sqrt{2 m E} / \hbar, \quad \sigma=\sqrt{2 m\left(E-V_{0}\right)} / \hbar
$$

and

$$
r_{c}(\epsilon)=(\epsilon-\sigma) /(\epsilon+\sigma), \quad t_{c}(\epsilon)=2 \epsilon /(\epsilon+\sigma) .
$$




\section{II.B PURE QUATERNIONIC POTENTIAL STEP}

By setting in Eq.(5) $V_{1}=0$ and $\sqrt{V_{2}^{2}+V_{3}^{2}}=V_{0}$, we obtain

$$
i \frac{\hbar^{2}}{2 m} \psi_{q}^{\prime \prime}(x)-j V_{0} \psi_{q}(x)=-\psi_{q}(x) E i
$$

The analytic plane wave solution for the pure quaternionic potential step reads

[I] FREE REGION : $\quad \psi_{q, I}(\epsilon, x)=\exp [i \epsilon x]+r_{q}(\epsilon) \exp [-i \epsilon x]+j \tilde{r}_{q}(\epsilon) \exp [\epsilon x]$,

[II] Potential Region : $\psi_{q, I I}(\epsilon, x)=(1+j w) t_{q}(\epsilon) \exp [i \rho x]+(\bar{w}+j) \tilde{t}_{q}(\epsilon) \exp [-\rho x]$,

where

$$
\epsilon=\sqrt{2 m E} / \hbar, \quad \rho=\sqrt{2 m \sqrt{E^{2}-V_{0}^{2}}} / \hbar, \quad w=-i V_{0} /\left(E+\sqrt{E^{2}-V_{0}^{2}}\right)
$$

and

$$
\begin{aligned}
r_{q}(\epsilon) & =(\epsilon-\rho) \exp [i \arctan (\epsilon / \rho)] / \sqrt{\epsilon^{2}+\rho^{2}}, \\
\tilde{r}_{q}(\epsilon) & =(1+i) \epsilon w /(\epsilon+\rho), \\
t_{q}(\epsilon) & =\epsilon / \rho, \\
\tilde{t}_{q}(\epsilon) & =\sqrt{\epsilon^{2}+\rho^{2}} \epsilon w \exp [-i \arctan (\rho / \epsilon)] /[\rho(\epsilon+\rho)] .
\end{aligned}
$$

For a detailed derivation of the plane wave solution for a quaternionic potential step, we refer the reader to the paper cited in ref. [1].

\section{WAVE PACKET ANALYSIS}

Until now, we have been concerned only with plane waves. In this section, we are going to study the time evolution of quaternionic wave packets and deducing from them several important results. The principle of superposition guarantees that every real linear combination of the plane waves in region I and region II,

$$
\Psi_{I, I I}(x, t)=\int \mathrm{d} \epsilon g(\epsilon) \psi_{I, I I}(\epsilon, x) \exp [-i E t / \hbar] \quad\left[E=(\hbar \epsilon)^{2} / 2 m\right],
$$

will satisfy the Schrödinger equation in the presence of a potential step. The reason why the use of the wave packet formalism is very interesting lies in the fact that the calculations, in the case of a gaussian convolution function

$$
g(\epsilon)=\exp \left[a^{2}\left(\epsilon-\epsilon_{0}^{2}\right) / 4\right] / 2 \sqrt{\pi},
$$

can be analytically approximated. This will allow to check and interpret our numerical results. In the next subsections, we first discuss the wave packets motion in the case of a standard (complex) potential step and, then, we analyze the special case of a pure quaternionic potential.

\section{III.A COMPLEX CASE}

The wave packets in region I and region II are given by

$$
\begin{aligned}
& \Omega_{c, I}(x, t)=\int_{a \epsilon_{\min }}^{+\infty} \mathrm{d}(a \epsilon) g(\epsilon)\left\{\exp [i \epsilon x]+r_{c}(\epsilon) \exp [-i \epsilon x]\right\} \exp \left[-i \epsilon^{2} \hbar t / 2 m\right], \\
& \Omega_{c, I I}(x, t)=\int_{a \epsilon_{\min }}^{+\infty} \mathrm{d}(a \epsilon) g(\epsilon)\left\{t_{c}(\epsilon) \exp [i \sigma x]\right\} \exp \left[-i \epsilon^{2} \hbar t / 2 m\right],
\end{aligned}
$$

where

$$
a \epsilon_{\min }=a \sqrt{2 m V_{0}} / \hbar .
$$


We find three wave packets: incident, reflected and transmitted,

$$
\begin{aligned}
& \Omega_{c, \text { inc }}(x, t)=\int_{a \epsilon_{\min }}^{+\infty} \mathrm{d}(a \epsilon) g(\epsilon) \exp \left[i\left(\epsilon x-\epsilon^{2} \hbar t / 2 m\right)\right], \\
& \Omega_{c, \text { ref }}(x, t)=\int_{a \epsilon_{\min }}^{+\infty} \mathrm{d}(a \epsilon) g(\epsilon) r_{c}(\epsilon) \exp \left[-i\left(\epsilon x+\epsilon^{2} \hbar t / 2 m\right)\right], \\
& \Omega_{c, \text { tra }}(x, t)=\int_{a \epsilon_{\min }}^{+\infty} \mathrm{d}(a \epsilon) g(\epsilon) t_{c}(\epsilon) \exp \left[i\left(\sigma x-\epsilon^{2} \hbar t / 2 m\right)\right] .
\end{aligned}
$$

The choice of a gaussian convolution function $g(\epsilon)$ peaked in $\epsilon_{0}\left(\epsilon_{0}>\epsilon_{\min }\right)$ and whose value is practically zero near to $\epsilon_{\min }$, i.e. $g\left(\epsilon_{\min }\right) \approx 0$, allows to legitimately approximate the incident wave packet as follows

$$
\begin{aligned}
\Omega_{c, \text { inc }}(x, t) & \approx \int_{-\infty}^{+\infty} \mathrm{d}(a \epsilon) g(\epsilon) \exp \left[i\left(\epsilon x-\epsilon^{2} \hbar t / 2 m\right)\right] \\
& =\exp \left[i\left(\epsilon_{0} x-a^{2} \epsilon_{0}^{2} \tau / 2\right)-i \arctan (2 \tau) / 2\right] \frac{\exp \left[-\left(x / a-a \epsilon_{0} \tau\right)^{2} /(1+2 i \tau)\right]}{\left(1+4 \tau^{2}\right)^{1 / 4}}
\end{aligned}
$$

where $\tau=\hbar t / m a^{2}$. In the case in which the variation of $r_{c}(\epsilon)$ can be neglected compared to that of $g(\epsilon)$, the reflected wave has the same form as the incident wave packet [11],

$$
\begin{aligned}
\Omega_{c, \text { ref }}(x, t) & \approx r_{c}\left(\epsilon_{0}\right) \int_{a \epsilon_{\min }}^{+\infty} \mathrm{d}(a \epsilon) g(\epsilon) \exp \left[-i\left(\epsilon x+\epsilon^{2} \hbar t / 2 m\right)\right] \\
& =r_{c}\left(\epsilon_{0}\right) \Omega_{c, \text { inc }}(-x, t) .
\end{aligned}
$$

For the transmitted wave packet, by neglecting the variation of $t_{c}(\epsilon)$ compared to that of $g(\epsilon)$ and by approximating $\sigma$ by its first order Taylor expansion, $\sigma_{0}+\left(\epsilon-\epsilon_{0}\right) \epsilon_{0} / \sigma_{0}$, we find

$$
\begin{aligned}
\Omega_{c, \text { tra }}(x, t) & \approx t_{c}\left(\epsilon_{0}\right) \exp \left[i\left(\sigma_{0}^{2}-\epsilon_{0}^{2}\right) x / \sigma_{0}\right] \int_{a \epsilon_{\min }}^{+\infty} \mathrm{d}(a \epsilon) g(\epsilon) \exp \left[i\left(\epsilon \epsilon_{0} x / \sigma_{0}-\epsilon^{2} \hbar t / 2 m\right)\right] \\
& =t_{c}\left(\epsilon_{0}\right) \exp \left[i\left(\sigma_{0}^{2}-\epsilon_{0}^{2}\right) x / \sigma_{0}\right] \Omega_{c, \text { inc }}\left(\epsilon_{0} x / \sigma_{0}, t\right) .
\end{aligned}
$$

The reflection probability (the ratio between the probabilities of finding the particle in the reflected packet, at a positive time $t_{0}$, and in the incident packet, at time $-t_{0}$ ) is given by

$$
\begin{aligned}
P_{c, \text { ref }} & =\int_{-\infty}^{0} \mathrm{~d} x\left|\Omega_{c, \text { ref }}\left(x, t_{0}\right)\right|^{2} / \int_{-\infty}^{0} \mathrm{~d} x\left|\Omega_{c, \text { inc }}\left(x,-t_{0}\right)\right|^{2} \\
& \approx\left|r_{c}\left(\epsilon_{0}\right)\right|^{2} \int_{-\infty}^{0} \mathrm{~d} x\left|\Omega_{c, \text { inc }}\left(-x, t_{0}\right)\right|^{2} / \int_{-\infty}^{0} \mathrm{~d} x\left|\Omega_{c, \text { inc }}\left(x,-t_{0}\right)\right|^{2} \\
& =\left|r_{c}\left(\epsilon_{0}\right)\right|^{2} .
\end{aligned}
$$

Similarly, the transmission probability is given by

$$
\begin{aligned}
P_{c, \text { tra }} & =\int_{0}^{+\infty} \mathrm{d} x\left|\Omega_{c, \text { tra }}\left(x, t_{0}\right)\right|^{2} / \int_{-\infty}^{0} \mathrm{~d} x\left|\Omega_{c, \text { inc }}\left(x,-t_{0}\right)\right|^{2} \\
& \approx\left|t_{c}\left(\epsilon_{0}\right)\right|^{2} \int_{0}^{+\infty} \mathrm{d} x\left|\Omega_{c, \text { inc }}\left(\epsilon_{0} x / \sigma_{0}, t_{0}\right)\right|^{2} / \int_{-\infty}^{0} \mathrm{~d} x\left|\Omega_{c, \text { inc }}\left(x,-t_{0}\right)\right|^{2} \\
& =\left|t_{c}\left(\epsilon_{0}\right)\right|^{2} \frac{\sigma_{0}}{\epsilon_{0}} \int_{0}^{+\infty} \mathrm{d} x\left|\Omega_{c, \text { inc }}\left(x, t_{0}\right)\right|^{2} / \int_{-\infty}^{0} \mathrm{~d} x\left|\Omega_{c, \text { inc }}\left(x,-t_{0}\right)\right|^{2} \\
& =\frac{\sigma_{0}}{\epsilon_{0}}\left|t_{c}\left(\epsilon_{0}\right)\right|^{2} .
\end{aligned}
$$

It is easy to verify that

$$
P_{c, \mathrm{ref}}+P_{c, \mathrm{tra}}=\left[\left(\frac{\epsilon-\sigma}{\epsilon+\sigma}\right)^{2}+\frac{\sigma}{\epsilon} \frac{4 \epsilon^{2}}{(\epsilon+\sigma)^{2}}\right]_{0}=1 .
$$




\section{III.B PURE QUATERNIONIC CASE}

The wave packets in region I and region II are given by

$$
\begin{aligned}
\Omega_{q, I}(x, t)= & \int_{a \epsilon_{\min }}^{+\infty} \mathrm{d}(a \epsilon) g(\epsilon)\left\{\exp [i \epsilon x]+r_{q}(\epsilon) \exp [-i \epsilon x]+j \tilde{r}_{q}(\epsilon) \exp [\epsilon x]\right\} \exp \left[-i \epsilon^{2} \hbar t / 2 m\right], \\
\Omega_{q, I I}(x, t)= & \int_{a \epsilon_{\min }}^{+\infty} \mathrm{d}(a \epsilon) g(\epsilon)\left\{t_{q}(\epsilon) \exp [i \rho x]+\bar{w} \tilde{t}_{q}(\epsilon) \exp [-\rho x]\right\} \exp \left[-i \epsilon^{2} \hbar t / 2 m\right]+ \\
& j \int_{a \epsilon_{\min }}^{\infty} \mathrm{d}(a \epsilon) g(\epsilon)\left\{w t_{q}(\epsilon) \exp [i \rho x]+\tilde{t}_{q}(\epsilon) \exp [-\rho x]\right\} \exp \left[-i \epsilon^{2} \hbar t / 2 m\right] .
\end{aligned}
$$

For sufficiently large negative $t$, only the (complex) incident wave packet

$$
\Omega_{q, \text { inc }}(x, t \ll 0)=\int_{a \epsilon_{\min }}^{+\infty} \mathrm{d}(a \epsilon) g(\epsilon) \exp \left[i\left(\epsilon x-\epsilon^{2} \hbar t / 2 m\right)\right]=\Omega_{c, \text { inc }}(x, t)
$$

exists. For sufficiently large positive $t$, the evanescent wave packets disappear and we only find one (complex) reflected wave packet,

$$
\Omega_{q, \mathrm{ref}}(x, t \gg 0)=\int_{a \epsilon_{\min }}^{+\infty} \mathrm{d}(a \epsilon) g(\epsilon) r_{q}(\epsilon) \exp \left[-i\left(\epsilon x+\epsilon^{2} \hbar t / 2 m\right)\right] \approx r_{q}\left(\epsilon_{0}\right) \Omega_{c, \text { inc }}(-x, t),
$$

and one (quaternionic) transmitted wave packet,

$$
\begin{aligned}
\Omega_{q, \mathrm{tra}}(x, t \gg 0) & =\int_{a \epsilon_{\min }}^{+\infty} \mathrm{d}(a \epsilon) g(\epsilon)(1+j w) t_{q}(\epsilon) \exp \left[i\left(\rho x-\epsilon^{2} \hbar t / 2 m\right)\right] \\
& \approx\left(1+j w_{0}\right) t_{q}\left(\epsilon_{0}\right) \exp \left[i\left(\rho_{0}^{4}-\epsilon_{0}^{4}\right) x / \rho_{0}^{3}\right] \Omega_{c, \text { inc }}\left(\epsilon_{0}^{3} x / \rho_{0}^{3}, t\right) .
\end{aligned}
$$

A simple calculation shows that

$$
P_{q, \text { ref }}=\int_{-\infty}^{0} \mathrm{~d} x\left|\Omega_{q, \text { ref }}\left(x, t_{0}\right)\right|^{2} / \int_{-\infty}^{0} \mathrm{~d} x\left|\Omega_{q, \text { inc }}\left(x,-t_{0}\right)\right|^{2} \approx\left|r_{q}\left(\epsilon_{0}\right)\right|^{2}
$$

and

$$
P_{q, \text { tra }}=\int_{0}^{+\infty} \mathrm{d} x\left|\Omega_{q, \text { tra }}\left(x, t_{0}\right)\right|^{2} / \int_{-\infty}^{0} \mathrm{~d} x\left|\Omega_{q, \text { inc }}\left(x,-t_{0}\right)\right|^{2} \approx \frac{\rho_{0}^{3}}{\epsilon_{0}^{3}}\left(1+\left|w_{0}\right|^{2}\right)\left|t_{q}\left(\epsilon_{0}\right)\right|^{2} .
$$

Finally,

$$
P_{q, \mathrm{ref}}+P_{q, \mathrm{tra}}=\left[\frac{(\epsilon-\rho)^{2}}{\epsilon^{2}+\rho^{2}}+\frac{\rho^{3}}{\epsilon^{3}}\left(1+\frac{\epsilon^{2}-\rho^{2}}{\epsilon^{2}+\rho^{2}}\right) \frac{\epsilon^{2}}{\rho^{2}}\right]_{0}=1
$$

\section{NUMERICAL RESULTS AND INTERPRETATION}

The results of our numerical study are plotted in Fig. 1, where the probability densities $\left|\Omega_{c}(x, t)\right|^{2}$ (complex case) and $\left|\Omega_{q}(x, t)\right|^{2}$ (pure quaternionic case) are drawn as a function of $x / a$ for different values of $\tau=\hbar t / m a^{2}$. The incident wave packets are centered in $E_{0}=2 V_{0}$ (diffusion) and the potential is given by $a \sqrt{2 m V_{0}}=10^{2} \hbar$. The data show an interesting phenomenon. For the same potential and the same incident energy, the reflected waves have different amplitudes in the case of a complex or a pure quaternionic potential step, and the transmitted waves move with different velocities. The quaternionic transmitted wave packet moves faster than the complex one. Let us try to understand these results by using the analytic approximations given in section III for the incident, reflected and transmitted wave packets and by using the fact that for small times, $\tau \ll 1$, no spreading effect is 
present. This allows to simplify the expressions given in section III for the complex and quaternionic wave packets. The amplitude of the incident wave is then given by

$$
\left|\Omega_{q, \text { inc }}(x, t)\right| \approx\left|\Omega_{c, \text { inc }}(x, t)\right| \approx \exp \left[-\left(x / a-a \epsilon_{0} \tau\right)^{2}\right]
$$

For the reflected waves, we have

$$
\begin{aligned}
\left|\Omega_{c, \mathrm{ref}}(x, t)\right| & \approx\left|r_{c}\left(\epsilon_{0}\right)\right| \exp \left[-\left(x / a+a \epsilon_{0} \tau\right)^{2}\right], \\
\left|\Omega_{q, \mathrm{ref}}(x, t)\right| & \approx\left|r_{q}\left(\epsilon_{0}\right)\right| \exp \left[-\left(x / a+a \epsilon_{0} \tau\right)^{2}\right] .
\end{aligned}
$$

This implies

$$
\begin{aligned}
& \left|\Omega_{c, \text { ref }}^{\max }(x, t)\right|^{2} /\left|\Omega_{c, \text { inc }}^{\max }(x, t)\right|^{2} \approx\left|r_{c}\left(\epsilon_{0}\right)\right|^{2} \quad \approx 2.94 \times 10^{-2}, \\
& \left|\Omega_{q, \text { ref }}^{\max }(x, t)\right|^{2} /\left|\Omega_{q, \text { inc }}^{\max }(x, t)\right|^{2} \approx\left|r_{q}\left(\epsilon_{0}\right)\right|^{2} \quad \approx 2.58 \times 10^{-3}, \\
& \left|\Omega_{c, \text { ref }}(x, t)\right|^{2} /\left|\Omega_{q, \text { ref }}(x, t)\right|^{2} \approx\left|r_{c}\left(\epsilon_{0}\right)\right|^{2} /\left|r_{q}\left(\epsilon_{0}\right)\right|^{2} \approx 11.4,
\end{aligned}
$$

which confirms the numerical results given in Fig.1 (negative $x$-axis). We can also see that the reflected waves move with the same velocity,

$$
v_{c, \text { ref }}=v_{q, \text { ref }}=-\hbar \epsilon_{0} / m .
$$

For the transmitted waves, by observing that

$$
\begin{aligned}
& \left|\Omega_{c, \text { tra }}(x, t)\right| \approx\left|t_{c}\left(\epsilon_{0}\right)\right| \exp \left[-\left(\epsilon_{0} / \sigma_{0}\right)^{2}\left(x / a-a \sigma_{0} \tau\right)^{2}\right], \\
& \left|\Omega_{q, \text { tra }}(x, t)\right| \approx\left|\left(1+j w_{0}\right) t_{q}\left(\epsilon_{0}\right)\right| \exp \left[-\left(\epsilon_{0} / \rho_{0}\right)^{6}\left(x / a-a \rho_{0}^{3} \tau / \epsilon_{0}^{2}\right)^{2}\right],
\end{aligned}
$$

we find that the centers of the complex and quaternionic transmitted wave packets move, respectively, with velocities

$$
v_{c, \text { tra }}=\hbar, \sigma_{0} / m \quad \text { and } \quad v_{q, \text { tra }}=\hbar \rho_{0}^{3} / m \epsilon_{0}^{2} .
$$

Consequently,

$$
\begin{aligned}
& \frac{x_{c, \mathrm{tra}}^{\max }(t)}{a}=\sqrt{\frac{E_{0}}{V_{0}}-1} \frac{a \sqrt{2 m V_{0}}}{\hbar} \frac{\hbar t}{m a^{2}}, \\
& \frac{x_{q, \mathrm{tax}}^{\max }(t)}{x_{c, \mathrm{tra}}^{\max }(t)}=\left[\left(\frac{E_{0}}{V_{0}}-1\right)\left(\frac{E_{0}}{V_{0}}+1\right)^{3}\left(\frac{V_{0}}{E_{0}}\right)^{4}\right]^{1 / 4},
\end{aligned}
$$

and by using

$$
E_{0}=2 V_{0}, \quad \frac{a \sqrt{2 m V_{0}}}{\hbar}=10^{2}, \quad \tau=\frac{\hbar t}{m a^{2}},
$$

we find

$$
\begin{aligned}
& \frac{x_{c, \text { tra }}^{\max }(\tau)}{a}=10^{2} \tau, \\
& \frac{x_{q, \text { tra }}^{\max }(\tau)}{x_{c, \text { tra }}^{\max }(\tau)} \approx 1.14 .
\end{aligned}
$$

This agrees with our numerical calculations as shown by the motion of the complex and quaternionic transmitted wave packets plotted in Fig. 1 (positive $x$-axis). For example, at time $\tau=0.15$ the maximum of the complex transmitted wave is found at $x=15 a$, whereas the center of the quaternionic wave packet (which moves faster) reaches at the same time the point $x \approx 17.1 \mathrm{a}$.

\section{CONCLUSIONS}

In the last years, the Schrödinger equation in the presence of quaternionic potentials with constant and space-dependent phase has been a matter of study and discussion in the literature [4-6]. Some properties of this class of potentials are detailed discussed in the Adler book [9] which represents a 
milestone in such a field. If quaternionic quantum mechanics represents a possible way to describe the nature, then it becomes relevant to examine how the predictions of standard theories may be affected by changing from complex to quaternionic potentials. The first theoretical analysis of quaternionic potential barriers was developped in $[2,3]$ and showed that in contrast with the standard complex case where the left and right transmission probability are equal in magnitude and phase, in the quaternionic quantum mechanics only the magnitude are equal. So, the measurement of a phase shift should be an indicator of quaternionic effects and space-dependent phase. Nevertheless, as remarked in [9], experiments to detect a phase shift are equivalent to detecting time-reversal violation and consequently cannot be seen in neutron-optical experiments [12-14]. A more complete phenomenology of the quaternionic potential barrier is given in [4]. With respect to previous works regarding potential barrier diffusion, in this paper, we have introduced the quaternionic wave packet formalism. This allow to study new qualitative differences between standard quantum mechanics and theoretical solutions obtained by solving the Schrödinger equation in the presence of a quaternionic step. For a detailed discussion of experimental proposals on quaternionic potentials and quantum mechanical systems in which a CP violation is interpreted by potentials with a space-dependent phase, we refer the reader to references $[1,4]$.

In this paper, we have seen that for incoming particles with an energy spectrum centered in $E_{0}=2 V_{0}$ the complex and quaternionic transmitted wave packets move with different velocities,

$$
\frac{v_{q, \text { tra }}}{v_{c, \text { tra }}}=\frac{\sqrt{3 \sqrt{3}}}{2} \text {. }
$$

Due to the fact that the complex and quaternionic incident wave packets coincide for large negative times (incoming particles), we could try to fit the motion of the quaternionic wave packet by using a different complex potential step. For example by choosing a new complex potential step, $W_{0}$, such that

$$
\sqrt{\frac{E_{0}}{W_{0}}-1}=\left[\left(\frac{E_{0}}{V_{0}}\right)^{2}-1\right]^{3 / 4} \frac{V_{0}}{E_{0}},
$$

we find $x_{q, \text { tra }}^{\max }(t)=x_{c, \text { tra }}^{\max }(t)$. In the particular case investigated in this paper, $E_{0}=2 V_{0}$, this implies

$$
\frac{E_{0}}{W_{0}}=1+\frac{3 \sqrt{3}}{4} .
$$

In this scenario, we have the same incident wave and complex or quaternionic transmitted wave packets which move with the same velocity (note that the reflected waves, for a same incoming energy spectrum, always move with the same velocity because propagate in the free potential region). Once we have guaranteed same velocities in the potential region, we have to check the probability of transmission (or equivalently the probability of reflection). A simple calculation (see section III) shows that

$$
\begin{aligned}
P_{c, \text { ref }} \approx & \left(\frac{\sqrt{E_{0}}-\sqrt{E_{0}-W_{0}}}{\sqrt{E_{0}}-\sqrt{E_{0}-W_{0}}}\right)^{2} \approx 2.01 \times 10^{-2}, \\
P_{q, \text { ref }} \approx & \frac{\left[\sqrt{E_{0}}-\left(E_{0}^{2}-V_{0}^{2}\right)^{1 / 4}\right]^{2}}{E_{0}-\sqrt{E_{0}^{2}-V_{0}^{2}}} \approx 2.58 \times 10^{-3} .
\end{aligned}
$$

This gives an interesting answer to the old question concerning the possibility to fit a quaternionic potential by a complex one and consequently to never recognize deviations from standard quantum mechanics. The study presented for a very simple potential, i.e. the potential step, shows that incoming particles with a given energy spectrum behave differently if they are diffused by a complex or a pure quaternionic potential step. This result, obtained by numerical calculations and interpreted by our gaussian analytical approximations, cannot be seen in the plane wave analysis. In such a limit, we only have reflection and transmission probabilities, and the quaternionic case can always be interpreted in terms of a complex potential step which appropriately fits the quaternionic probabilities. This paper stimulates further investigations, for example it should be interesting to study, within the wave packet formalism, diffusion and tunnelling by complex and pure quaternionic potential barrier which, surely, represents a more realistic potential to be tested by an experimental analysis. In the case 
of the potential barrier, it is of great interest to examine the tunnelling phenomena, in particular, in a forthcoming paper we aim to analyze in detail the Hartman effect $[15,16]$ in the presence of a pure quaternioic potential. The possibility to treat the potential barrier problem as a two-step problem $[7,8]$ gives a more solid interest to this paper, what it could appear an academic quantum mechanical exercise play a fundamental role in the understanding the behavior of wave packets in the presence of a more complicate potential. The analysis done in this paper explicitly shows that a qualitative difference exists between complex and quaternionic quantum mechanics.

\section{References}

1. S. De Leo, G. C. Ducati and T. M. Madureira, "Analytic plane wave solutions for the quaternionic potential step", J. Math. Phys. A 47, 082106-15 (2006).

2. A. J. Davies and B. H. McKellar, "Non-relativistic quaternionic quantum mechanics", Phys. Rev. A 40, 4209-4214 (1989).

3. A. J. Davies and B. H. McKellar, "Observability of quaternionic quantum mechanics", Phys. Rev. A 46, 3671-3675 (1992).

4. S. De Leo, G. C. Ducati and C. C. Nishi, "Quaternionic potential in non-relativistic quantum mechanics", J. Phys. A 35, 5411-5426 (2002).

5. S. De Leo and G. C. Ducati, "Quaternionic bound states", J. Phys. A 38, 3443-3454 (2005).

6. S. De Leo and G. C. Ducati, "Quaternionic diffusion by a potential step", J. Math. Phys. A 47, 102104-9 (2006).

7. A. Bernardini, S. De Leo and P. Rotelli, "Above barrier potential diffusion", Mod. Phys. Lett. A 19, $2717-2725$ (2004).

8. S. De Leo and P. Rotelli, "Above barrier Dirac multiple scattering and resonances", Eur. Phys. J. C 46, 551-558 (2006).

9. S. L. Adler, Quaternionic quantum mechanics and quantum fields, (New York: Oxford University Press, 1995).

10. S. De Leo and G. C. Ducati , "Quaternionic differential operators", J. Math. Phys. 42, 2236-2265 (2001).

11. C. Choen-Tannoudji, B. Diu and F. Lalöe, Quantum mechanics, (New York: John Wiley \& Sons, 1977).

12. A. Peres, "Proposed test for complex versus quaternion quantum mechanics", Phys. Rev. Lett. 42, 683-686 (1979).

13. H. Kaiser, E. A. George and S. A. Werner, "Neutron interferometric search for quaternions in quantum mechanics", Phys. Rev. A 29, 2276-2279 (1984).

14. A. G. Klein, "Schrödinger inviolate: neutron optical serches for violations of quantum mechanics", Physica B 151, 44-49 (1989).

15. T.E. Hartman, "Tunnelling of a wave packet", J. Appl. Phys. 33, 3427-3432 (1962).

16. V.S. Olkhovsky, E. Recami, and J, Jakiel, "Unified time analysis of photon and particle tunnelling", Phys. Rep. 398, 133-178 (2004). 


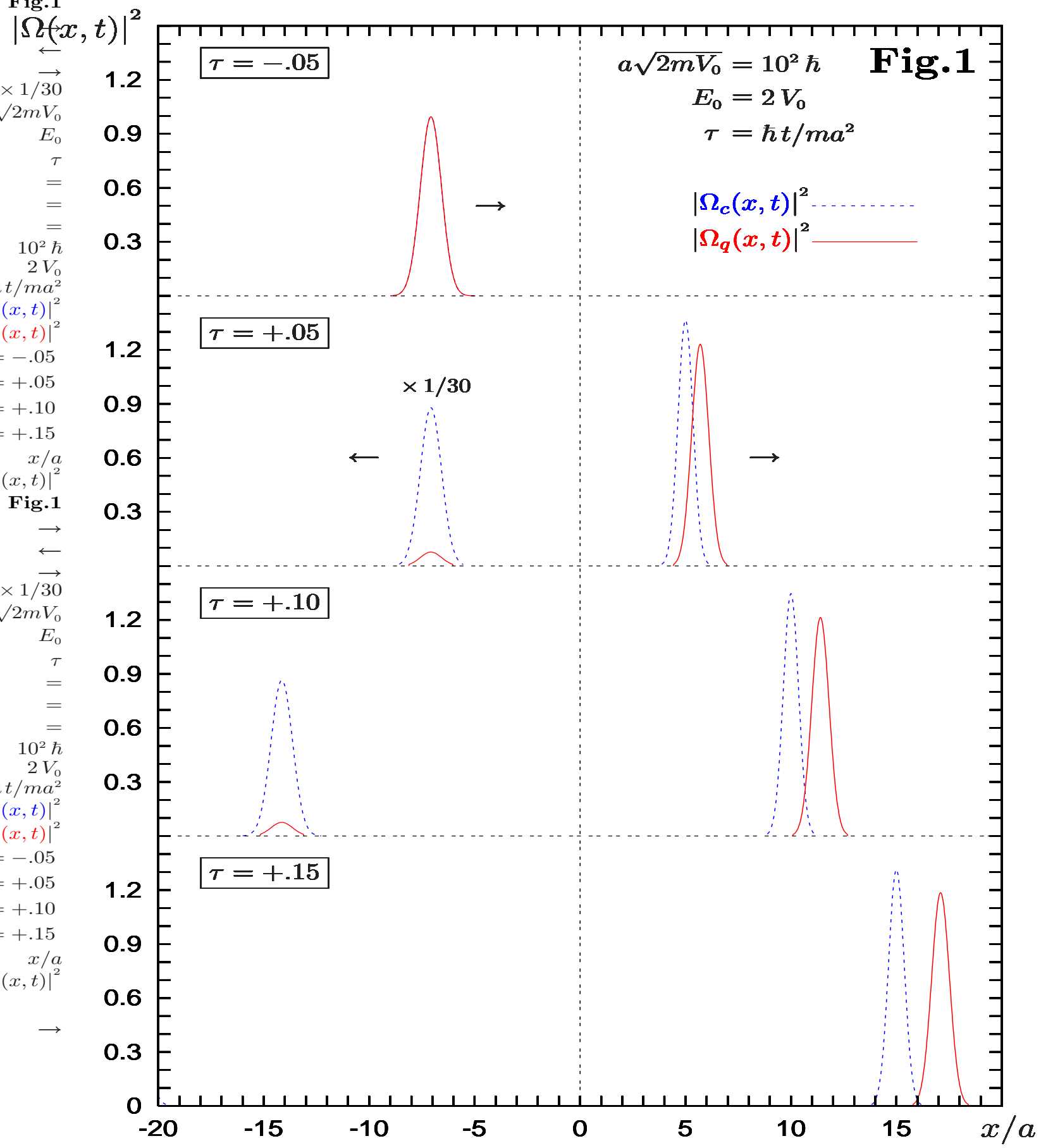

Fig. 1. Diffusion $\left(E_{0}=2 V_{0}\right)$ of a wave packet at a complex and pure quaternionic potential step. The complex $\left(V_{0}=V_{1}\right)$ and quaternionic $\left(V_{0}=\sqrt{V_{2}^{2}+V_{2}^{2}}\right)$ potentials act for positive $x$. For negative time $(\tau<0)$ only the incident packet is present and it moves towards the step. After a certain time, we find four packets. The reflected packets, $\left|\Omega_{c, \text { inc }}(x, t)\right|^{2}$ and $\left|\Omega_{q \text {,inc }}(x, t)\right|^{2}$, are returning to the left moving with the same velocity. The transmitted packets, $\left|\Omega_{c, \text { tra }}(x, t)\right|^{2}$ and $\left|\Omega_{q, \text { tra }}(x, t)\right|^{2}$, propagate towards the right and move with different velocities. 